\title{
Changes in lipoprotein lipase and endothelial lipase mass in familial hypercholesterolemia during three-drug lipid-lowering combination therapy
}

Hayato Tada ${ }^{1 *}$, Junji Kobayashi ${ }^{2}$, Masa-aki Kawashiri ${ }^{1}$, Kazuya Miyashita $^{3}$, Atsushi Nohara $^{4}$, Akihiro Inazu $^{5}$, Katsuyuki Nakajima ${ }^{6}$, Hiroshi Mabuchi ${ }^{4}$ and Masakazu Yamagishi ${ }^{1}$

\begin{abstract}
Background: This study was performed to compare the effects of three different lipid-lowering therapies (statins, ezetimibe, and colestimide) on lipoprotein lipase and endothelial lipase masses in pre-heparin plasma (pre-heparin $L P L$ and EL mass, respectively) from patients with familial hypercholesterolemia (FH). FH is usually treated by coadministration of these three drugs.

Methods: The pre-heparin LPL and EL masses were measured in fresh frozen plasma drawn and stored at various time points during coadministration of the three drugs from patients with heterozygous FH harboring a single mutation in the LDL receptor ( $n=16$, mean age 63 years). The patients were randomly divided into two groups based on the timing when ezetimibe was added.

Results: Plasma $L P L$ mass concentration was significantly reduced by rosuvastatin at $20 \mathrm{mg} /$ day (median $=87.4$ [IQR: 71.4-124.7] to 67.5 [IQR: 62.1-114.3] ng/ml, $P<0.05$ ). In contrast, ezetimibe at $10 \mathrm{mg} / \mathrm{day}$ as well as colestimide at $3.62 \mathrm{~g} /$ day did not alter its level substantially (median $=67.5$ [IQR: 62.1-114.3] to 70.2 [IQR: 58.3-106.2], and to 74.9 [IQR: $55.6-101.3] \mathrm{ng} / \mathrm{ml}$, respectively) in the group starting with rosuvastatin followed by the addition of ezetimibe and colestimide. On the other hand, the magnitude in LPL mass reduction was lower in the group starting with ezetimibe at $10 \mathrm{mg} /$ day before reaching the maximum dose of $20 \mathrm{mg} /$ day of rosuvastatin. Plasma EL mass concentration was significantly increased by rosuvastatin at $20 \mathrm{mg} /$ day (median $=278.8$ [IQR: 186.7-288.7] to 297.0 [IQR: $266.2-300.2] \mathrm{ng} / \mathrm{ml}, P<0.05)$, whereas other drugs did not significantly alter its level.

Conclusion: The effects on changes of LPL and EL mass differed depending on the lipid-lowering therapy, which may impact the prevention of atherosclerosis differently.
\end{abstract}

Keywords: Lipoprotein lipase, Endothelial lipase, Familial hypercholesterolemia

\section{Background}

Lipoprotein lipase $(L P L)$ and endothelial lipase $(E L)$ hydrolyze triglyceride (TG) in circulating chylomicrons and very low-density lipoprotein (VLDL) on the surface of endothelial cells [1]. It has been demonstrated that a reduced concentration of plasma $L P L$ mass is associated with an increased risk of coronary artery disease $[2,3]$.

\footnotetext{
*Correspondence: ht240z@sa3.so-net.ne.jp

'Division of Cardiovascular Medicine, Kanazawa University Graduate School

of Medicine, 13-1 Takara-machi, Kanazawa 920-8641, Japan

Full list of author information is available at the end of the article
}

Several studies have demonstrated that plasma $L P L$ mass concentration could be altered by drug manipulations, such as fibrate, insulin sensitizer, and statins in patients with diabetes, potentially affecting the progression of their atherosclerosis [4-6]. In addition, recent genetic studies have suggested that genetic modulators of $L P L$ mediate cardiovascular risk. For example, loss-of-function mutations in apolipoprotein $\mathrm{C} 3$ (APOC3), which is an inhibitor of $L P L$, decrease the risk of coronary artery disease (CAD) [7], while loss-of-function mutations in apolipoprotein A5 (APOA5), which is an activator of $L P L$, increases the risk 
of CAD [8]. In contrast, large-scale clinical trials assessing the efficacy of HDL cholesterol-raising therapies have failed $[9,10]$ based on the neutral impact of HDL cholesterol concentration on CAD estimated by Mendelian randomization studies [11, 12]. Accordingly, targeting plasma $L P L$ (and $E L$ ) concentrations, rather than plasma HDL cholesterol concentration, could be much more reasonable in terms of preventive cardiology. However, no study has yet assessed the changes in plasma lipase mass concentration during lipid-lowering therapy in patients with familial hypercholesterolemia (FH), which usually requires the coadministration of different types of such drugs [13]. Therefore, we compared the effects of three different lipid-lowering therapies (statins, ezetimibe, and colestimide) on $L P L$ and $E L$ mass in pre-heparin plasma (pre-heparin $L P L$ and $E L$ mass, respectively) in patients with $\mathrm{FH}$.

\section{Methods}

\section{Study subjects}

The study population consisted of patients with heterozygous $\mathrm{FH}$ who participated in a previous clinical trial conducted as a prospective open randomized study to investigate the efficacy and safety of coadministration of rosuvastatin $(20 \mathrm{mg} /$ day $)$, ezetimibe $(10 \mathrm{mg} /$ day $)$, and granulated colestimide (3.62 g/day) at the maximum doses permitted in Japan. All 17 subjects were heterozygous with a confirmed LDL receptor gene mutation and fulfilled our clinical diagnostic criteria for heterozygous FH: patients with primary hyper-LDL cholesterolemia $(>160 \mathrm{mg} / \mathrm{dl})$ with tendon xanthoma or those with firstdegree relatives with previously diagnosed heterozygous $\mathrm{FH}$ showing primary hyper-LDL cholesterolemia ( $>160 \mathrm{mg} / \mathrm{dl})$. Exclusion criteria of the present study were $\mathrm{FH}$ patients with a homozygous gene mutation, patients under LDL apheresis therapy or any immunomodulatory medication, patients with fasting serum triglyceride levels $>500 \mathrm{mg} / \mathrm{dl}$, patients with hepatic disease, or patients within 12 weeks after the onset of an acute myocardial infarction or stroke. Details of this study have been described elsewhere [14], and 16 of 17 patients whose fresh frozen plasma was available were included in this study. The subjects were divided into two groups using a sealed envelope-based method according to the timing when ezetimibe was added at $10 \mathrm{mg} /$ day. All participants were started on a 4week treatment with rosuvastatin at $5 \mathrm{mg} /$ day followed by another 4-week treatment of rosuvastatin at $10 \mathrm{mg} /$ day. The dose of rosuvastatin in group 1 was increased to $20 \mathrm{mg}$ with an 8-week follow-up, whereas group 2 received ezetimibe at $10 \mathrm{mg} /$ day with an 8-week follow-up (phase 1). After phase 1, group 1 received ezetimibe at $10 \mathrm{mg} /$ day added to rosuvastatin for 8 weeks, whereas in group 2 the doses of rosuvastatin were increased to $20 \mathrm{mg}$ with an 8-week follow-up (phase 2). In phase 3, groups 1 and 2 were given $3.62 \mathrm{~g}$ of granulated colestimide (twice a day before meals, once in the morning and once in the evening) added to the phase 2 treatment regimen (Fig. 1).

\section{Determination of $L P L$ and $E L$ mass concentrations}

Fasting blood samples were drawn and stored at $-80{ }^{\circ} \mathrm{C}$ during the study period. Pre-heparin $L P L$ concentrations were determined with a highly sensitive and specific ELISA kit (Code No.27184; IBL, Fujioka, Japan) $[15,16]$.

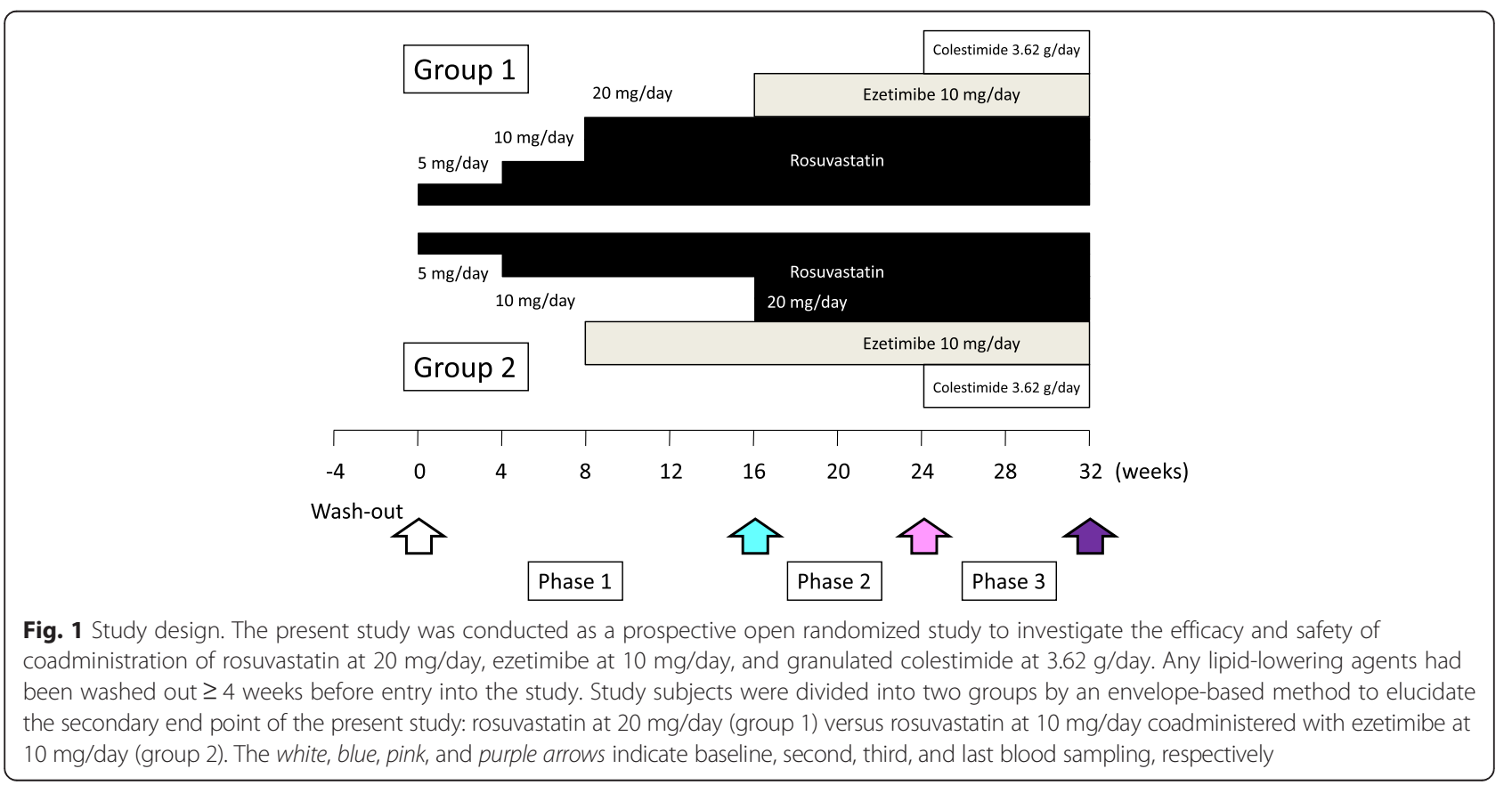


$E L$ mass concentrations were determined using our ELISA system (Code No. 27182; IBL) [17].

\section{Biochemical analysis}

Fasting blood samples were drawn for assays. Plasma concentrations of total cholesterol, TG, and HDL cholesterol were determined enzymatically as described previously [18]. Remnant-like particles (RLP) were determined as described previously [19].

\section{Statistical analysis}

Categorical variables are expressed as percentages. Continuous variables with a normal distribution are shown as means $( \pm S D)$, and those with a skewed distribution are shown as medians (interquartile [IQR]). The changes in each lipase mass concentration were compared by analysis of variance (ANOVA). All statistical analyses were conducted using $\mathrm{R}$. In all analyses, $P<0.05$ was assumed to indicate statistical significance.

\section{Ethical considerations}

This study was approved by the Ethics Committees of Kanazawa University (Number: 1883-1) and Kanazawa Medical University (Number: 272). All procedures were performed in accordance with the ethical standards of the responsible committee on human experimentation (institutional and national) and with the Helsinki Declaration of 1975, as revised in 2008. Informed consent for genetic analyses was obtained from subjects with $\mathrm{FH}$ for inclusion in the study.

\section{Results}

\section{Baseline characteristics of study subjects}

Sixteen Japanese subjects with heterozygous FH were enrolled in the present study. Baseline characteristics and concomitant drug therapies are listed in Table 1. Five of six diabetic patients (28\%) that were under hypoglycemic medical therapy had a glycohemoglobin concentration $<7.0 \%$. No patients were treated with insulin injection therapy. Dosages of coadministered medications were kept constant during the entire study period.

\section{Changes in LPL mass during coadministration of three drugs}

In group 1 , the $L P L$ mass concentration was significantly reduced by rosuvastatin at $20 \mathrm{mg} /$ day $($ median $=87.4$ [IQR: 71.4-124.7] to 67.5 [IQR: 62.1-.3] ng/ml, $P<0.05$, Fig. 2a), whereas ezetimibe at $10 \mathrm{mg} /$ day as well as colestimide at $3.62 \mathrm{~g} /$ day did not markedly alter its level (median =67.5 [IQR: 62.1-114.3] to 70.2 [IQR: 58.3106.2], and to 74.9 [IQR: $55.6-101.3$ ] ng/ml, respectively, $P=$ NS, Fig. 2a). Similar trends were observed in group 2 , in which the statistical significance of the reduction
Table 1 Baseline characteristics

\begin{tabular}{|c|c|c|c|}
\hline & All $(n=16)$ & Group $1(n=9)$ & Group $2(n=7)$ \\
\hline Age (yr) & $63 \pm 10$ & $60 \pm 11$ & $68 \pm 7$ \\
\hline Male $(n, \%)$ & $11(69 \%)$ & $4(44 \%)$ & $5(71 \%)$ \\
\hline Body mass index $\left(\mathrm{kg} / \mathrm{m}^{2}\right)$ & $23.7 \pm 2.0$ & $23.1 \pm 1.9$ & $24.5 \pm 1.9$ \\
\hline Total cholesterol (mg/dl) & $380 \pm 42$ & $394 \pm 56$ & $364 \pm 51$ \\
\hline Triglyceride (mg/dl) & 95 [84-127] & $95[88-126]$ & $91[68-136]$ \\
\hline HDL cholesterol (mg/dl) & $46 \pm 10$ & $49 \pm 14$ & $43 \pm 6$ \\
\hline LDL cholesterol (mg/dl) & $299 \pm 48$ & $309 \pm 46$ & $283 \pm 48$ \\
\hline RLP cholesterol (mg/dl) & $8.9 \pm 3.3$ & $8.7 \pm 3.5$ & $9.2 \pm 3.0$ \\
\hline Hypertension (n, \%) & $8(50 \%)$ & $3(33 \%)$ & $5(71 \%)$ \\
\hline Diabetes (n, \%) & $6(38 \%)$ & $3(33 \%)$ & $3(43 \%)$ \\
\hline Smoking (n, \%) & $1(6 \%)$ & $0(0 \%)$ & $1(14 \%)$ \\
\hline $\begin{array}{l}\text { Coronary artery } \\
\text { disease }(n, \%)\end{array}$ & $9(56 \%)$ & $4(44 \%)$ & $5(71 \%)$ \\
\hline
\end{tabular}

$R L P$ remnant-like particle

during phase 1 was diminished using combination therapy consisting of rosuvastatin at $10 \mathrm{mg} /$ day and ezetimibe at $10 \mathrm{mg} /$ day (median $=79.3$ [IQR: 58.8-85.0] to 77.5 [IQR: $60.0-84.6$ ] ng/ml, $P=$ NS, Fig. 3a). On the other hand, the significant reduction in LPL mass concentration achieved by adding colestimide at $3.62 \mathrm{~g} /$ day was replicated in group 2 (median $=81.9$ [IQR: 47.987.0] to 75.1 [IQR: $43.3-85.5$ ] ng/ml, $P<0.05$, Fig. 3a).

\section{Changes in EL mass during coadministration of three drugs}

In group 1, the $E L$ mass concentration was significantly increased by rosuvastatin at $20 \mathrm{mg} /$ day (median $=278.8$ [IQR: 186.7-288.7] to 297 [IQR: 266.2-300.2] ng/ml, $P$ $<0.05$, Fig. 2b), whereas ezetimibe at $10 \mathrm{mg} /$ day as well as colestimide at $3.62 \mathrm{~g} /$ day did not markedly change its level $($ median $=297$ [IQR: 266.2-300.2] to 241.8 [IQR: 232.0-305.6], and to 243.5 [IQR: 231.6-298.5] ng/ml, respectively, $P=$ NS, Fig. $2 b$ ). In group 2, combination therapy with rosuvastatin at $10 \mathrm{mg} /$ day and ezetimibe at $10 \mathrm{mg} /$ day also increased its level significantly (median $=250.6$ [IQR: $175.6-351.8$ ] to 300.3 [IQR: 238.0375.0] ng/ml, $P<0.05$, Fig. 3b). On the other hand, there were no significant changes in EL mass concentration when ezetimibe was added at $10 \mathrm{mg} /$ day and colestimide at $3.62 \mathrm{~g} /$ day (median $=300.3$ [IQR: 238.0375.0 ] to 369.2 [IQR: 212.0-451.2], and to 296.3 [IQR: 231.7-424.1] ng/ml, respectively, $P=$ NS, Fig. $3 b)$.

\section{Association between plasma lipids and lipase mass}

We investigated the associations between changes in $L P L / E L$ and those in LDL cholesterol, TG, or RLP cholesterol during each phase. There were no significant associations between the changes in LPL mass concentrations and those in lipids (Additional file 1: Figures S1-S6). 

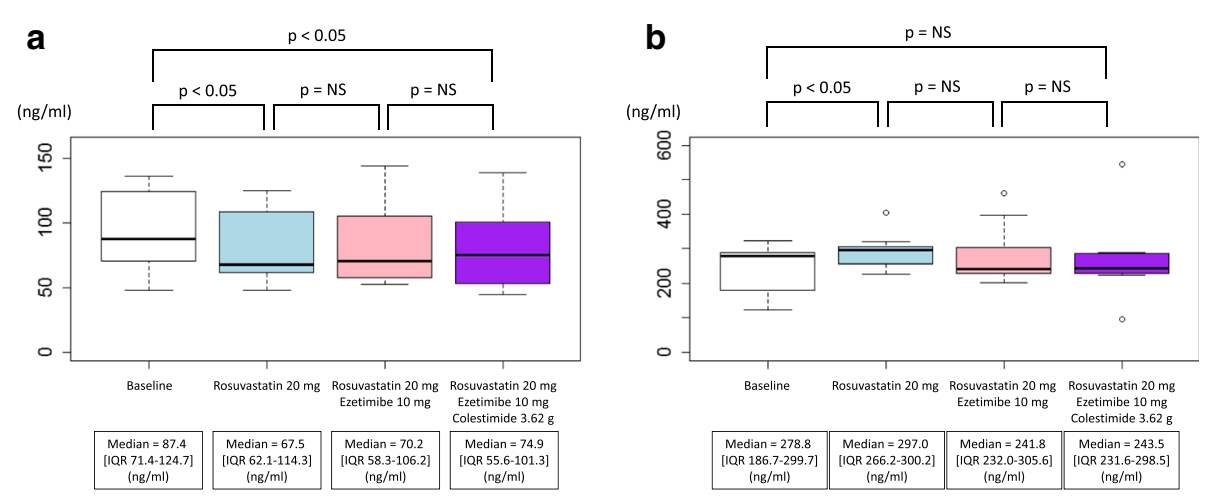

Fig. 2 Lipase mass concentration during coadministration of three drugs in group 1. a LPL mass concentration. $\mathbf{b} E L$ mass concentration. White indicates the baseline. Blue indicates the second blood sampling when rosuvastatin was administered at $20 \mathrm{mg} /$ day. Pink indicates the third blood sampling when rosuvastatin at $20 \mathrm{mg} /$ day and ezetimibe at $10 \mathrm{mg} /$ day were coadministered. Purple indicates the last blood sampling when rosuvastatin at $20 \mathrm{mg} /$ day, ezetimibe at $10 \mathrm{mg} /$ day, and colestimide at $3.62 \mathrm{~g} /$ day were coadministered

Significant associations between the changes in $E L$ mass concentrations and TG/RLP cholesterol were observed during phase 2 in group 2 (Additional file 1: Figures S7-S12).

\section{Discussion}

We measured the pre-heparin $L P L$ and $E L$ mass at each time point during coadministration of three drugs (statin, ezetimibe, and colestimide) in patients with heterozygous FH harboring a single mutation in the LDL receptor to compare the effects of these drugs on the plasma lipase mass concentration. Our results indicate that (1) statin and colestimide significantly reduced plasma $L P L$ mass, but ezetimibe did not alter its level; (2) statin significantly increased plasma $E L$ mass, but ezetimibe and colestimide did not change its level; (3) there was no clear association between changes in lipase mass and changes in plasma lipid levels.
Recent Mendelian randomization trials have suggested that plasma TG is one of the causal factors for the development of coronary artery disease, rather than merely a marker [7, 20]. In addition, plasma TG level is one of the residual risks in this statin era [21]. Accordingly, reassessment of TG-rich lipoprotein metabolism seems to be a reasonable strategy to combat such residual risk. In this regard, genetic studies have indicated that the $L P L$ and $A P O C 3$ pathway is strongly associated with plasma TG as well as coronary artery disease, gathering a great deal of attention as a novel therapeutic target. Increasing plasma $L P L$ concentration as well as reducing plasma $A P O C 3$, rather than increasing plasma HDL cholesterol, could be beneficial targets.

The results of one study that investigated the effect of statins on changes in $L P L$ mass concentration in patients with diabetes showed results that were contrary to ours [6]. This difference may have been because we used the
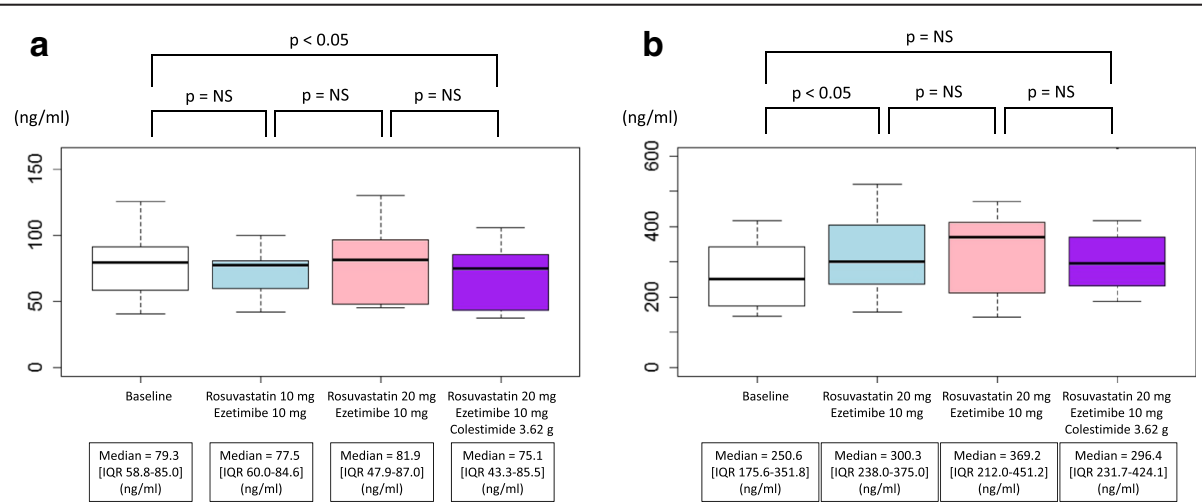

Fig. 3 Lipase mass concentration during coadministration of three drugs in group 2. a LPL mass concentration. $\mathbf{b} E L$ mass concentration. White indicates the baseline. Blue indicates the second blood sampling when rosuvastatin at $10 \mathrm{mg} /$ day and ezetimibe at $10 \mathrm{mg} /$ day were coadministered. Pink indicates the third blood sampling when rosuvastatin at $20 \mathrm{mg} /$ day and ezetimibe at $10 \mathrm{mg} / \mathrm{day}$ were coadministered. Purple indicates the last blood sampling when rosuvastatin at $20 \mathrm{mg} /$ day, ezetimibe at $10 \mathrm{mg} /$ day, and colestimide at $3.62 \mathrm{~g} /$ day were coadministered 
maximum dose of rosuvastatin in patients with $\mathrm{FH}$ whose TG-rich lipoprotein levels were shown to be impaired.

Our study has several limitations. First, the size of the study population was small because of the rarity of the disease (diagnosed genetic heterozygous FH). However, we observed similar tendencies between the two groups divided according to the timing of the initiation of ezetimibe. Second, this study did not have a crossover design, which could potentially lead to biased assessment of the differences among drugs. Third, we measured pre-heparin lipase mass levels instead of post-heparin levels, which could potentially affect the results, especially $L P L$ mass levels.

\section{Conclusion}

In conclusion, the effects on changes of $L P L$ and $E L$ mass were different depending on the lipid-lowering therapy, which may have different impacts on the preventive effects of the therapy on atherosclerosis.

\section{Additional file}

Additional file 1: Associations between the changes in LPL/EL mass and those in lipids. (PPTX $2501 \mathrm{~kb}$ )

\section{Abbreviations \\ ANOVA: Analysis of variance; APOA5: Apolipoprotein A5; \\ APOC3: Apolipoprotein C3; CAD: Coronary artery disease; EL: Endothelial lipase; FH: Familial hypercholesterolemia; IQR: Interquartile; LPL: Lipoprotein lipase; RLP: Remnant-like particle; TG: Triglyceride; VLDL: Very low-density lipoprotein.}

\section{Competing interests}

Hayato Tada has received research grants from Banyu Life Science Foundation International, SENSHIN Medical Research Foundation, The Uehara Memorial Foundation, Takeda Science Foundation, Astellas Atherosclerosis Update, and Mochida Memorial Foundation. Junji Kobayashi has received research grants from Pfizer Inc. and Kowa Company, Ltd.

\section{Authors' contributions}

HT participated in the collection and analysis of data and writing of the manuscript. MK, KM, AN, Al, KN, HM, and MY participated in data collection and analysis. JK participated in conception of the study, supervision, data analysis and manuscript editing. All authors read and approved the final version of the manuscript.

\section{Acknowledgments}

We are grateful to Kazuko Honda and Sachio Yamamoto (staff of Kanazawa University) for their outstanding technical assistance.

\section{Sources of funding}

This work was supported by a scientific research grant from the Ministry of Education (grant number 15 K08627).

\section{Author details}

${ }^{1}$ Division of Cardiovascular Medicine, Kanazawa University Graduate School of Medicine, 13-1 Takara-machi, Kanazawa 920-8641, Japan. '2Department of General Medicine, Kanazawa Medical University, Uchinada, Japan. ${ }^{3}$ Immuno-Biological Laboratories Co., Ltd, Fujioka, Japan. ${ }^{4}$ Department of Advanced Research in Community Medicine, Kanazawa University Graduate School of Medical Science, Kanazawa, Japan. ${ }^{5}$ Department of Laboratory Science, Molecular Biochemistry and Molecular Biology, Graduate School of Medical Science, Kanazawa University, Kanazawa, Japan. ${ }^{6}$ Department of Laboratory Sciences, Gunma University Graduate School of Health Sciences, Maebashi, Japan.
Received: 3 March 2016 Accepted: 29 March 2016

Published online: 02 April 2016

\section{References}

1. Nilsson-Ehle P, Garfinkel AS, Schotz MC. Lipolytic enzymes and plasma lipoprotein metabolism. Annu Rev Biochem. 1980;49:667-93.

2. Hitsumoto T, Ohsawa H, Uchi T, Noike H, Kanai M, Yoshinuma M, et al. Preheparin serum lipoprotein lipase mass is negatively related to coronary atherosclerosis. Atherosclerosis. 2000;153:391-6.

3. Hitsumoto T, Yoshinaga K, Aoyagi K, Sakurai T, Kanai M, Uchi T, et al. Association between preheparin serum lipoprotein lipase mass and acute myocardial infarction in Japanese men. J Atheroscler Thromb. 2002;9:163-9.

4. Totsuka M, Miyashita Y, Ito Y, Watanabe H, Murano T, Shirai K. Enhancement of preheparin serum lipoprotein lipase mass by bezafibrate administration. Atherosclerosis. 2000;153:175-9.

5. Shirai $K$, Itoh $Y$, Sasaki H, Totsuka M, Murano T, Watanabe $H$, et al. The effect of insulin sensitizer, troglitazone, on lipoprotein lipase mass in preheparin serum. Diabetes Res Clin Pract. 1999;46:35-41.

6. Endo K, Miyashita Y, Saiki A, Oyama T, Koide N, Ozaki H, et al. Atorvastatin and pravastatin elevated pre-heparin lipoprotein lipase mass of type 2 diabetes with hypercholesterolemia. J Atheroscler Thromb. 2004;11:341-7.

7. TG and HDL Working Group of the Exome Sequencing Project, National Heart, Lung, and Blood Institute, Crosby J, Peloso GM, et al. Loss-of-function mutations in APOC3, triglycerides, and coronary disease. N Engl J Med. 2014;371:22-31.

8. Do R, Stitziel NO, Won HH, Jørgensen AB, Duga S, Angelica Merlini $P$, et al. Exome sequencing identifies rare LDLR and APOA5 alleles conferring risk for myocardial infarction. Nature. 2015;518:102-6.

9. AIM-HIGH Investigators, Boden WE, Probstfield JL, Anderson T, Chaitman BR, Desvignes-Nickens $P$, et al. Niacin in patients with low $\mathrm{HDL}$ cholesterol levels receiving intensive statin therapy. N Engl J Med. 2011;365:2255-67.

10. Schwartz GG, Olsson AG, Abt M, Ballantyne CM, Barter PJ, Brumm J, et al. Effects of dalcetrapib in patients with a recent acute coronary syndrome. $\mathrm{N}$ Engl J Med. 2012;367:2089-99.

11. Voight BF, Peloso GM, Orho-Melander M, Frikke-Schmidt R, Barbalic M, Jensen MK, et al. Plasma HDL cholesterol and risk of myocardial infarction: a Mendelian randomisation study. Lancet. 2012;380:572-80.

12. Do R, Willer CJ, Schmidt EM, Sengupta S, Gao C, Peloso GM, et al. Common variants associated with plasma triglycerides and risk for coronary artery disease. Nat Genet. 2013:45:1345-52.

13. Gidding SS, Ann Champagne M, de Ferranti SD, Defesche J, Ito MK, Knowles JW, et al. The agenda for familial hypercholesterolemia: A scientific statement from the American Heart Association. Circulation. 2015;132:2167-92

14. Kawashiri MA, Nohara A, Noguchi T, Tada H, Nakanishi C, Mori M, et al. Efficacy and safety of coadministration of rosuvastatin, ezetimibe, and colestimide in heterozygous familial hypercholesterolemia. Am J Cardiol. 2012;109:364-9.

15. Shirakawa T, Nakajima K, Shimomura Y, Kobayashi J, Stanhope K, Havel P, et al. Comparison of the effect of post-heparin and pre-heparin lipoprotein lipase and hepatic triglyceride lipase on remnant lipoprotein metabolism. Clin Chim Acta. 2015:440:193-200.

16. Shirakawa T, Nakajima K, Yatsuzuka S, Shimomura Y, Kobayashi J, Machida $T$, et al. The role of circulating lipoprotein lipase and adiponectin on the particle size of remnant lipoproteins in patients with diabetes mellitus and metabolic syndrome. Clin Chim Acta. 2015;440:123-32.

17. Ishida T, Miyashita K, Shimizu M, Kinoshita N, Mori K, Sun L, et al. ELISA system for human endothelial lipase. Clin Chem. 2012;58:1656-64.

18. Tada H, Kawashiri MA, Takata M, Matsunami K, Imamura A, Matsuyama M, et al. Infantile cases of sitosterolaemia with novel mutations in the ABCG5 gene: extreme hypercholesterolaemia is exacerbated by breastfeeding. JIMD Rep. 2015;21:115-22.

19. Tada H, Kawashiri MA, Tanaka A, Nakano T, Nakajima K, Inoue T, et al. Post-prandial remnant lipoprotein metabolism in autosomal recessive hypercholesterolaemia. Eur J Clin Invest. 2012;42:1094-9. 
20. CARDloGRAMplusC4D Consortium, Deloukas P, Kanoni S, Willenborg C, Farrall M, Assimes TL, et al. Large-scale association analysis identifies new risk loci for coronary artery disease. Nat Genet. 2013;45:25-33.

21. Schwartz GG, Abt M, Bao W, DeMicco D, Kallend D, Miller M, et al.

Fasting triglycerides predict recurrent ischemic events in patients with acute coronary syndrome treated with statins. J Am Coll Cardiol. 2015;65:2267-75

Submit your next manuscript to BioMed Central and we will help you at every step:

- We accept pre-submission inquiries

- Our selector tool helps you to find the most relevant journal

- We provide round the clock customer support

- Convenient online submission

- Thorough peer review

- Inclusion in PubMed and all major indexing services

- Maximum visibility for your research

Submit your manuscript at www.biomedcentral.com/submit 